\title{
Probing Muonphilic Force Carriers and Dark Matter at Kaon Factories
}

\author{
Gordan Krnjaic ${ }_{0},{ }^{1}$ Gustavo Marques-Tavares, ${ }^{2,3}$ Diego Redigolo, ${ }^{4,5,6}$ and Kohsaku Tobioka ${ }^{7,8}$ \\ ${ }^{1}$ Fermi National Accelerator Laboratory, Batavia, Illinois 60510, USA \\ ${ }^{2}$ Maryland Center for Fundamental Physics, Department of Physics, University of Maryland, College Park, Maryland 20742, USA \\ ${ }^{3}$ Stanford Institute for Theoretical Physics, Stanford University, Stanford, California 94305, USA \\ ${ }^{4}$ Tel-Aviv University, Tel-Aviv 6997801, Israel \\ ${ }^{5}$ Institute for Advanced Study, Princeton, New Jersey 08540, USA \\ ${ }^{6}$ Weizmann Institute of Science, Rehovot 7610001, Israel \\ ${ }^{7}$ Florida State University, Tallahassee, Florida FL 32306, USA \\ ${ }^{8}$ High Energy Accelerator Research Organization (KEK), Tsukuba 305-0801, Japan
}

(Received 8 May 2019; published 28 January 2020)

\begin{abstract}
Rare kaon decays are excellent probes of light, new weakly coupled particles. If such particles $X$ couple preferentially to muons, they can be produced in $K \rightarrow \mu \nu X$ decays. We evaluate the future sensitivity for this process at NA62 assuming $X$ decays either invisibly or to dimuons. Our main physics target is the parameter space that resolves the $(g-2)_{\mu}$ anomaly, where $X$ is a gauged $L_{\mu}-L_{\tau}$ vector or a muonphilic scalar. The same parameter space can also accommodate dark matter freeze-out or reduce the tension between cosmological and local measurements of $H_{0}$ if the new force decays to dark matter or neutrinos, respectively. We show that for invisible $X$ decays, a dedicated single muon trigger analysis at NA62 could probe much of the remaining $(g-2)_{\mu}$ favored parameter space. Alternatively, if $X$ decays to muons, NA62 can perform a dimuon resonance search in $K \rightarrow 3 \mu \nu$ events and greatly improve existing coverage for this process. Independently of its sensitivity to new particles, we find that NA62 is also sensitive to the standard model predicted rate for $K \rightarrow 3 \mu \nu$, which has never been measured.
\end{abstract}

DOI: 10.1103/PhysRevLett.124.041802

Introduction.-Light weakly coupled forces arise in many compelling extensions of the standard model (SM) and are the focus of a broad experimental effort [1-3]. If the corresponding force carriers (we refer to force carriers as "forces" throughout) couple preferentially to muons, they offer the last viable opportunity to resolve the long-standing $\sim 3.5 \sigma$ anomaly in $(g-2)_{\mu}$ [4-6] with new physics below the electroweak scale as proposed in Ref. [7]. (Light new particles with appreciable couplings to the first generation have been excluded in simple models, including both visibly and invisibly decaying dark photons; see Refs. $[3,8])$. Thus, there is strong motivation to improve experimental sensitivity to these interactions.

Furthermore, there is abundant evidence for the existence of dark matter (DM), whose microscopic properties remain elusive [9]. One possible explanation for these null results is that DM couples more strongly to the second and third generation. Indeed, there are several consistent, viable, and predictive dark forces which mediate DM freeze-out to higher generation particles [10,11]. Since muonic

Published by the American Physical Society under the terms of the Creative Commons Attribution 4.0 International license. Further distribution of this work must maintain attribution to the author(s) and the published article's title, journal citation, and DOI. Funded by SCOAP ${ }^{3}$. forces do not couple directly to first generation particles, these DM candidates are difficult to probe with direct detection experiments, but can be efficiently produced at accelerators.

It is known that muonic forces lead to new rare kaon decays [12-14]. However, there are several timely reasons to revisit this subject.

(1) The NA62 experiment [15] is currently producing unprecedented numbers of kaons, and is poised to considerably improve sensitivity to muonic forces.

(2) The $g-2$ Collaboration [16] and the J-PARC $g-2$ experiment [17] will soon decisively test the $(g-2)_{\mu}$ anomaly. If this discrepancy is due to new physics, the particles responsible necessarily predict SM deviations in other, complementary muonic systems.

(3) Recently there has been great interest in new proposals for dedicated experiments to probe muonic forces [11,18-21]. To assess the merits of these ideas, it is essential to know what existing experiments can achieve.

In this Letter we show that existing kaon factories can probe $K \rightarrow \mu \nu X$ decays, where $X$ is a new particle that couples preferentially to muons. Our main focus is the new physics opportunities of the NA62 experiment at CERN [22], which will produce $10^{13} \mathrm{~K}^{+}$. 
If $X$ decays invisibly, we find that, with a dedicated single muon trigger, NA62 could have unprecedented sensitivity to $K \rightarrow \mu \nu X(X \rightarrow$ invisible $)$ processes. Such a search could probe nearly all the remaining parameter space in which muonic forces reconcile the $(g-2)_{\mu}$ anomaly. If the invisible particles are DM, this also enables $X$-mediated thermal freeze-out [11]; if, instead, these particles are neutrinos, this same parameter space can ease the $\sim 3.5 \sigma$ tension in Hubble constant measurements [23].

If $X$ decays to muons, we find that an NA62 dimuon resonance search in $K \rightarrow \mu \nu X\left(X \rightarrow \mu^{+} \mu^{-}\right)$processes could greatly improve the coverage for both scalar and vector forces, thereby covering nearly all of the $(g-2)_{\mu}$ favored region for $m_{K}-m_{\mu}>m_{X}>2 m_{\mu}$. The irreducible background for this search arises from $K \rightarrow 3 \mu \nu$ decays which have never been observed before; intriguingly, we find that NA62 can also measure this process in existing data.

Vector forces.-Gauged $L_{\mu}-L_{\tau}$ : A vector $V$ gauging a spontaneously broken $L_{\mu}-L_{\tau}$ symmetry is a minimal candidate to explain the $(g-2)_{\mu}$ anomaly. The Lagrangian contains

$$
\mathcal{L} \supset \frac{m_{V}^{2}}{2} V_{\mu} V^{\mu}+V_{\mu}\left(g_{V} J_{V}^{\mu}+\epsilon e J_{\mathrm{EM}}^{\mu}\right),
$$

where $g_{V}$ is the gauge coupling, $m_{V}$ is the mass, and $J_{V}^{\mu}$ is the $L_{\mu}-L_{\tau}$ current [24]. Loops of taus and muons induce kinetic mixing with the photon $\epsilon \simeq g_{V} / 67$, which also couples $V$ to the electromagnetic (EM) current $J_{\mathrm{EM}}^{\mu}$ in Eq. (1). The widths for $V \rightarrow f \bar{f}$ are

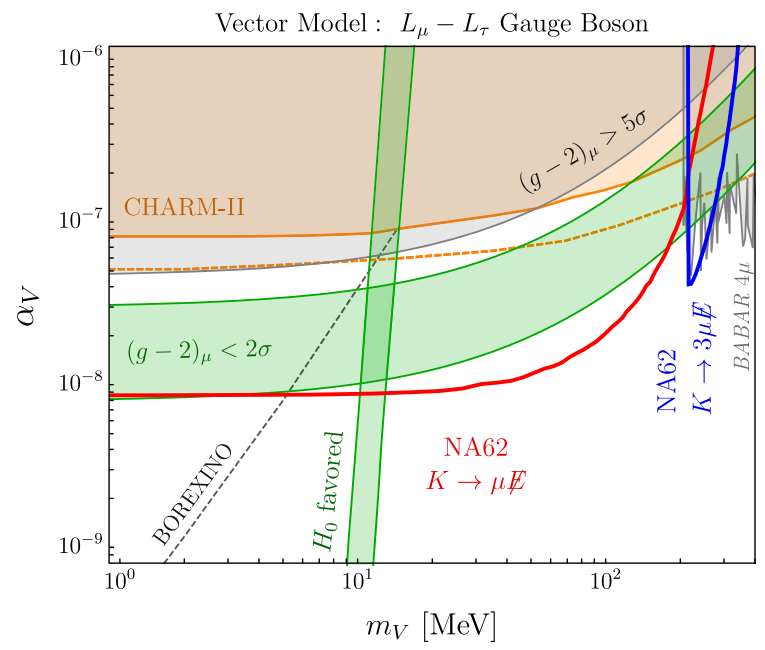

$$
\Gamma_{V \rightarrow f \bar{f}}=\frac{\alpha_{V} m_{V}}{3}\left(1+\frac{2 m_{\mu}^{2}}{m_{V}^{2}}\right) \sqrt{1-\frac{4 m_{\mu}^{2}}{m_{V}^{2}}},
$$

where $f=\mu, \tau$ and $\alpha_{V} \equiv g_{V}^{2} / 4 \pi$, and the width to neutrino flavor $\nu_{f}$ is $\Gamma_{V \rightarrow \nu_{f} \bar{\nu}_{f}}=\alpha_{V} m_{V} / 6$. Decays through the EM current are suppressed by additional factors of $\epsilon^{2} \alpha / \alpha_{V}$, so we neglect these here. In all of the parameter space we consider here, $V$ decays promptly within the $65 \mathrm{~m}$ decay region of NA62.

Although we require $m_{V} \gtrsim 1 \mathrm{MeV}$ to avoid tension with cosmology [25], for $m_{V} \sim$ few $\mathrm{MeV}, V \rightarrow \nu \bar{\nu}$ decays after neutrino decoupling increase the effective number of neutrino species by $\Delta N_{\text {eff }} \sim 0.2-0.5$, which can ameliorate the tension in Hubble rate measurements [23]; lighter masses are disfavored [26,27].

As shown in Fig. 1 (left), the NA62 $K \rightarrow \mu \nu X$ reach with $X$ decaying invisibly could cover a large portion of the parameter space, far beyond the reach of present experiments. Conversely, the $K \rightarrow \mu \nu X$ search with $X \rightarrow \mu \mu$ is competitive with $B A B A R$. The detailed study and the experimental challenges of the invisible and dimuon analyses are described in the two parts of the section on rare kaon decays at NA62, respectively.

Adding $L_{\mu}-L_{\tau}$ charged dark matter: If DM couples to $V, V \rightarrow$ DM decays can significantly change the $V$ branching fraction above the dimuon threshold; below this boundary, $V$ always decays invisibly (either to neutrinos or DM). Here we add a DM candidate $\chi\left(m_{V}>2 m_{\chi}\right)$

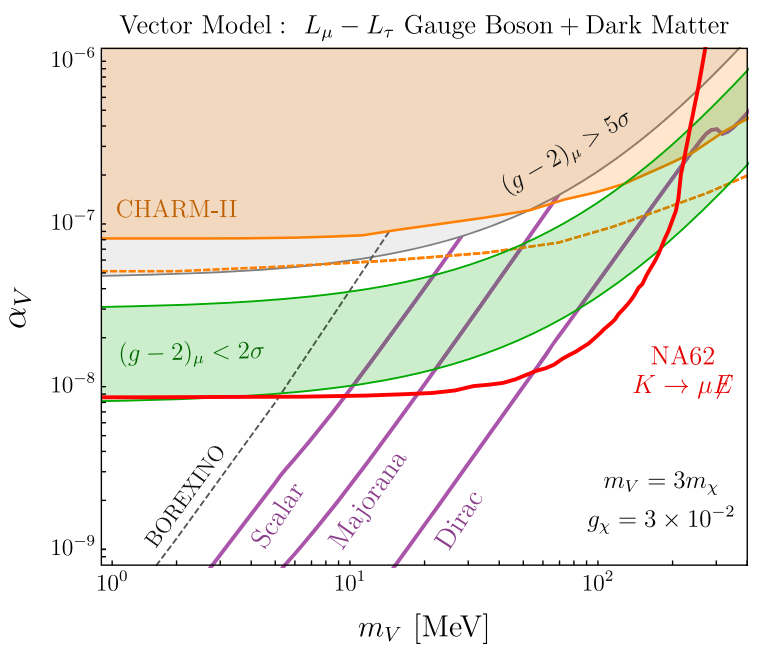

FIG. 1. Left: Parameter space for an $L_{\mu}-L_{\tau}$ SM extension from the vector forces section. The light green band is the $2 \sigma$ region accommodating the $(g-2)_{\mu}$ anomaly, while the green vertical region increases $\Delta N_{\text {eff }}=0.2-0.5$, ameliorating the $H_{0}$ tension [23]. We show projections for an NA62 search for $K^{+} \rightarrow \mu^{+} \nu_{\mu} V$ followed by a prompt invisible $V \rightarrow \nu \bar{\nu}$ decay (red curve) or a prompt visible $V \rightarrow \mu^{+} \mu^{-}$decay (blue curve). Both sensitivities assume the full NA62 luminosity to be recorded by the single muon and dimuon trigger, respectively, and systematic errors comparable to the statistical uncertainty (see the section on rare kaon decays at NA62 and the Supplemental Material [28] which contains Refs. [12,18,29-34] for details). We also show bounds from $B A B A R 4 \mu$, [35], $(g-2)_{\mu}$, and CHARM-II $\nu$ [36,37]; the dashed curve is the CCFR bound [38]. The dashed Borexino bound [19,39,40] assumes the mixing from SM loops. Right: Same as left, only the $V$ decays to dark matter $\chi$, with $\operatorname{BR}(V \rightarrow \chi \chi) \simeq 1$; the purple bands yield the observed $\mathrm{DM}$ abundance via freeze-out. 
charged under $L_{\mu}-L_{\tau}$ and extend Eq. (1) to include a coupling to the dark current $\mathcal{L} \supset g_{\chi} V_{\mu} J_{\chi}^{\mu}$. We now have

$$
J_{\chi}^{\mu}= \begin{cases}i \chi^{*} \partial_{\mu} \chi+\text { H.c. } & \text { complex scalar } \\ \frac{1}{2} \bar{\chi} \gamma^{\mu} \gamma^{5} \chi & \text { Majorana } \\ \bar{\chi} \gamma^{\mu} \chi & \text { Dirac, }\end{cases}
$$

where $g_{\chi} \equiv g_{V} q_{\chi}$ is the DM- $V$ coupling and $q_{\chi}$ is the DM $L_{\mu}-L_{\tau}$ charger; we assume $\mu, \tau$ and $\nu_{\mu, \tau}$ carry unit charge. For $m_{\chi}<m_{V}$, freeze-out proceeds via $s$-channel annihilation to SM particles for each model in Eq. (3) [11,41]. Figure 1 shows DM production targets alongside various constraints.

Scalar forces. - The minimal Lagrangian for a Yukawa muonic force is

$$
\mathcal{L}=\frac{1}{2}\left(\partial_{\mu} \phi\right)^{2}-\frac{m_{\phi}^{2}}{2} \phi^{2}-y_{\phi} \phi \bar{\mu} \mu,
$$

where $\phi$ is a real scalar particle. The interaction in Eq. (4) can arise, for instance, by integrating out a heavy, vectorlike lepton singlets whose mass mixes with the righthanded muon as discussed in the Supplemental Material [28]. In the absence of additional interactions, for $m_{\phi}>2 m_{\mu}$, the dominant decay is $\phi \rightarrow \mu^{+} \mu^{-}$, with partial width

$$
\Gamma_{\phi \rightarrow \mu^{+} \mu^{-}}=\frac{\alpha_{\phi} m_{\phi}}{2}\left(1-\frac{4 m_{\mu}^{2}}{m_{\phi}^{2}}\right)^{3 / 2},
$$

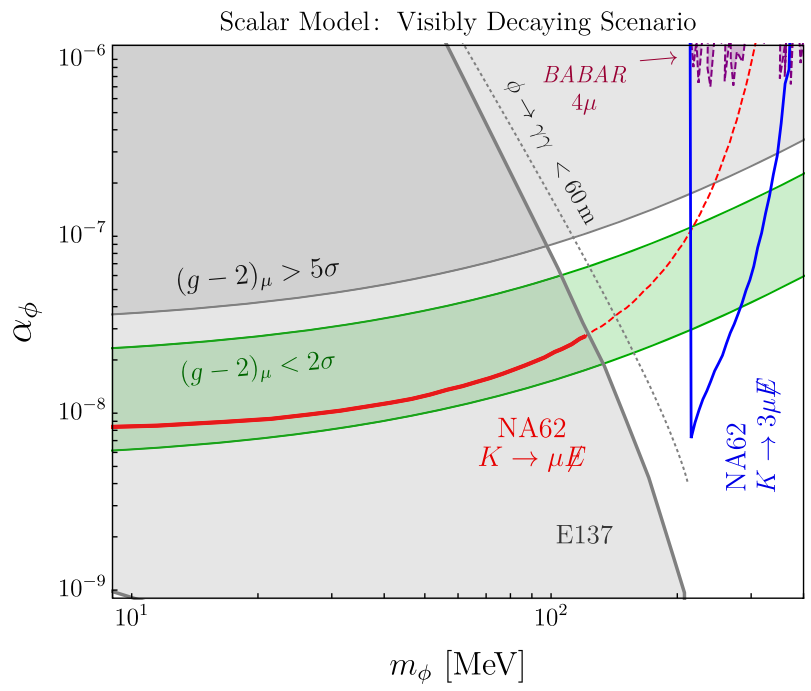

where $\alpha_{\phi} \equiv y_{\phi}^{2} / 4 \pi$. For $m_{\phi}<2 m_{\mu}$, the dominant channel is $\phi \rightarrow \gamma \gamma$ through a muon loop with width

$$
\Gamma_{\phi \rightarrow \gamma \gamma}=\frac{\alpha_{\mathrm{EM}}^{2} \alpha_{\phi} m_{\phi}^{3}}{64 \pi^{2} m_{\mu}^{2}}\left|\frac{2}{x^{2}}\left[x+(x-1) \arcsin ^{2} \sqrt{x}\right]\right|^{2},
$$

where $x \equiv m_{\phi}^{2} / 4 m_{\mu}^{2}$ and the lab frame decay length is

$\ell_{\phi \rightarrow \gamma \gamma} \sim 60 \mathrm{~m}\left(\frac{3 \times 10^{-6}}{\alpha_{\phi}}\right)\left(\frac{50 \mathrm{MeV}}{m_{\phi}}\right)^{4}\left(\frac{E_{\phi}}{75 \mathrm{GeV}}\right)$,

where the $m_{\phi}^{-4}$ scaling accounts for the boost factor. In this minimal "visibly decaying" scenario, most of our favored parameter space is below the dimuon threshold, so the diphoton channel dominates and, for the maximum $\phi$ energy $\sim 75 \mathrm{GeV}$, nearly all decays occur outside the NA62 detector to mimic a missing energy signature. However, a dedicated study is required to identify the distance beyond which these decays are invisible given NA62 kinematics and acceptance; we also note that it may be possible to perform a $\phi \rightarrow \gamma \gamma$ resonance search if this occurs inside the decay region.

Alternatively, $\phi$ may decay predominantly to undetected particles (e.g., DM) in the "invisibly decaying" scenario. In both cases, the scalar is produced via $K \rightarrow \mu \nu \phi$ processes whose width is computed in the Supplemental Material [28].

Figure 2 shows the NA62 projections for visible (left) and invisible (right) decays assuming $100 \%$ branching ratio in both channels. The main difference relative to the vector

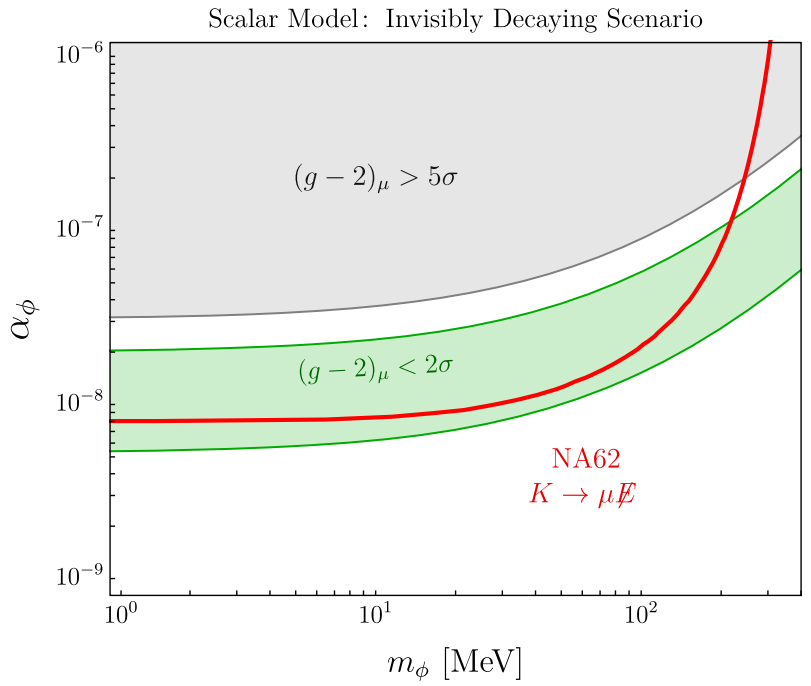

FIG. 2. Parameter space and NA62 projection for a muonphilic scalar particle $\phi$ described in the scalar forces section. Here we define $\alpha_{\phi} \equiv y_{\phi}^{2} / 4 \pi$, where $y_{\phi}$ is the Yukawa coupling to muons from Eq. (4) and the light green band accommodates the $(g-2)_{\mu}$ anomaly. Left: Projections for an NA62 search for $K^{+} \rightarrow \mu^{+} \nu_{\mu} \phi$, where $\phi$ decays visibly into $\phi \rightarrow \mu^{+} \mu^{-}$or $\gamma \gamma$ where kinematically allowed. On the left of the dashed gray line the lifetime of the muonphilic scalar is long enough to give an invisible signal at NA62. Also shown are E137 constraints from Ref. [42]. Right: Same as the left, but $\phi$ decays invisibly. Both assume the full NA62 luminosity and the searches to be statistics dominated (see the section on rare kaon decays at NA62 and Supplemental Material [28] for details). 
case is that the $K \rightarrow 3 \mu \nu$ search improves considerably beyond the BABAR $4 \mu$ bounds; here the $e^{+} e^{-} \rightarrow \mu^{+} \mu^{-} \phi$ cross section is much smaller than $V$ production. We also show the E137 bound for visible decays from Ref. [42] (see also Ref. [43]). There are additional constraints from supernovae $[42,44]$ not included in the figure due to their large astrophysical uncertainties and significant model dependence in the invisible decaying scenario.

Rare kaon decays at NA62.-The electroweak coupling governing SM $K \rightarrow \mu \nu$ decays is

$$
\mathcal{L} \supset\left(2 G_{F} f_{K} V_{u s}\right) \partial_{\alpha} K^{-} \bar{\nu}_{\mu} \gamma^{\alpha} P_{L} \mu+\text { H.c. },
$$

where $G_{F}$ is the Fermi constant, $V_{u s}=0.223$ is the $u s$ Cabibbo Kobayashi Maskawa (CKM) element, and $f_{K}=$ $160 \mathrm{MeV}$ is the kaon decay constant. We are interested in three-body corrections to this process: $K^{+} \rightarrow \mu^{+} \nu_{\mu} X$, where $X=V$ or $\phi$ is emitted from a final state $\mu$ and/or $\nu_{\mu}$ line. The differential decay distribution is

$$
\frac{d \Gamma\left(K^{+} \rightarrow \mu^{+} \nu X\right)}{d m_{\mathrm{miss}}^{2}}=\frac{1}{256 \pi^{3} m_{K}^{3}} \int \sum|\mathcal{M}|^{2} d m_{\mu X}^{2},
$$

where $m_{\mu X}$ is the $\mu X$ invariant mass and

$$
m_{\text {miss }}^{2} \equiv\left(P_{X}+P_{\nu_{\mu}}\right)^{2}=\left(P_{K}-P_{\mu}\right)^{2} .
$$

The matrix element $|\mathcal{M}|^{2}$ for both scenarios is calculated in the Supplemental Material [28]. Below we describe two different search strategies depending on whether $\mathrm{X}$ decays invisibly or to muons.

Invisible analysis:If $X$ is produced in $K^{+} \rightarrow \mu^{+} \nu_{\mu} X$ events and decays invisibly, the $m_{\text {miss }}^{2}$ distribution in $K \rightarrow \mu+$ invisible decays differs from the SM prediction (see Supplemental Material [28]). The sensitivity of an $m_{\text {miss }}^{2}$ search in single muon events is computed using the log-likelihood ratio

$$
\Lambda(S)=\sum_{i}-2 \log \frac{L_{i}(S)}{L_{i}(\hat{S}=0)},
$$

where $L_{i}$, the likelihood in each bin $i$, is constructed from a Poisson distribution [45], and $S=N_{K^{+}} \mathcal{A B R}\left(K^{+} \rightarrow\right.$ $\left.\mu^{+} \nu X\right)$ is the signal yield with acceptance $\mathcal{A} \simeq 0.35$. We require $\Lambda(S)<4$ to define the $2 \sigma$ sensitivity.

Our background sample is extracted from public NA62 data from the 2015 run in which $2.4 \times 10^{7}$ events passing the single muon trigger were recorded [31]. These data yield $N_{K^{+}} \approx 10^{8}$ kaons after dividing out the detector acceptance and SM branching ratio $\operatorname{BR}\left(K^{+} \rightarrow \mu^{+} \nu_{\mu}\right)=$ 0.63 ; all events in this sample are binned in missing mass intervals of $4 \times 10^{-3} \mathrm{GeV}^{2}$.

One of the main backgrounds for this search is $K \rightarrow \mu \nu(\gamma)$, in which a radiated $\gamma$ is not detected and contributes to the missing energy. This process peaks at $m_{\text {miss }}^{2}=0$ and its contribution to the large missing mass tail depends on NA62's photon rejection efficiency. Because of this large background, including missing mass bins below $m_{\text {miss }}^{2}=2.3 \times 10^{-2} \mathrm{GeV}^{2}$ does not change the log-likelihood ratio defined in Eq. (11).

In the 2015 data sample, other backgrounds are present at large $m_{\text {miss }}^{2}$ and exceed the $K \rightarrow \mu \nu(\gamma)$ tail for $m_{\text {miss }}^{2}>0.1 \mathrm{GeV}^{2}$. These events are largely due to the muon halo and we expect their contribution to be substantially reduced in the 2017 dataset where NA62 utilizes a silicon pixel detector (GTK) to measure the timing and momentum of upstream kaons [22]. To approximately account for this existing improvement, we rescale the background yield above $m_{\text {miss }}^{2}>2.3 \times 10^{-2} \mathrm{GeV}^{2}$ by an additional factor of 2 to estimate our sensitivity. For more details regarding our analysis and the challenges of maximizing signal sensitivity, see Supplemental Material [28], where we show how our results vary under different assumptions regarding systematic errors.

Dimuon analysis:If $X$ is produced in $K^{+} \rightarrow \mu^{+} \nu_{\mu} X$ events and decays visibly to dimuons, NA62 can improve upon previous experiments in the $K^{+} \rightarrow 2 \mu^{+} \mu^{-} \nu$ channel. The $\mathrm{SM}$ prediction is $\mathrm{BR}\left(K^{+} \rightarrow 2 \mu^{+} \mu^{-} \nu\right)_{\mathrm{SM}}=1.3 \times 10^{-8}$ [46] and currently has not been observed; the best limit comes from $\operatorname{E787} \mathrm{BR}\left(K^{+} \rightarrow 2 \mu^{+} \mu^{-} \nu\right)_{\text {obs }}<4.1 \times 10^{-7}$ in 1989 [47]. With current luminosity $\left(\sim 10^{11} K^{+}\right.$[30]), NA62 should already have recorded at least 100 such events passing the dimuon trigger. Here we propose a dimuon resonance search in $K^{+} \rightarrow \mu^{+} \nu X\left(\mu^{+} \mu^{-}\right)$events with opposite sign dimuon pairs.

Since these data have not been released yet by NA62, we estimate the sensitivity of the search from our MC simulation. We implement the effective weak interaction of Eq. (8), the electromagnetic interactions of $K^{+}$decays, and the new physics couplings from Eqs. (1) and (4) in MadGraph 5 v2 LO [48,49]. We neglect a subdominant contribution from a contact interaction of $K^{+} \mu^{+} \nu \gamma$. Both the background and the signal in $2 \mu^{+} \mu^{-} \nu$ final state are simulated. In Figs. 1 (left) and 2 (left) we present the results of this analysis in blue curves labeled NA62 $K \rightarrow 3 \mu \not E$. Systematic uncertainties on the background will affect less the result compared to the invisible channel because a data-driven background estimate would be possible. For more details about our projection, see supplementary material [28].

Conclusion.-In this Letter we have shown that rare kaon decay searches at NA62 can probe most of the remaining parameter space for which muonic-philic particles resolve the $\sim 3.5 \sigma(g-2)_{\mu}$ anomaly; these are the only viable explanations involving particles below the weak scale. The same parameter space can also accommodate thermal DM production or reduce the $H_{0}$ tension if the new particle decays to DM or neutrinos, respectively. 
If this new particle decays invisibly, achieving this sensitivity requires a dedicated single muon trigger to record all $K^{+} \rightarrow \mu^{+}+$invisible events with $m_{\text {miss }}^{2}>$ $0.05 \mathrm{GeV}^{2}$ during run 3 . The ultimate reach in this channel depends crucially on the systematic uncertainties on events with these kinematics; a dedicated experimental study is needed to assess the feasibility of this requirement.

We note that if the $(g-2)_{\mu}$ anomaly is confirmed, NA62 can play a key role in deciphering the new physics responsible for the discrepancy. However, even if future measurements are consistent with the SM, the searches we propose can still constrain models for which muonic forces mediate dark matter freeze-out. Such measurements can also inform future decisions about proposed dedicated experiments including $\mathrm{NA} 64 \mu$ [21], $\mathrm{M}^{3}$ [11], BDX [50,51], and LDMX [52,53].

We are grateful to Todd Adams, Wolfgang Altmannshofer, Gaia Lanfranchi, Roberta Volpe, and Yiming Zhong for helpful discussions and to Babette Döbrich and Evgueni Goudzovski for correspondence about NA62. We also thank Evgueni Goudzovski, Roberta Volpe, and Yiming Zhong for useful feedback on a preliminary version of the manuscript. Fermilab is operated by Fermi Research Alliance, LLC, under Contract No. DE-AC02-07CH11359 with the U.S. Department of Energy. G. M.-T. is supported by DOE Grant No. DESC0012012, NSF Grant No. PHY-1620074, and by the Maryland Center for Fundamental Physics. K. T. is supported by his start-up fund at Florida State University (Project No. 084011-550-042584). The authors thank the KITP where this work was initiated and the support of the National Science Foundation under Grant No. NSF PHY1748958.

[1] R. Essig et al., in Proceedings, 2013 Community Summer Study on the Future of U.S. Particle Physics: Snowmass on the Mississippi (CSS2013): Minneapolis, 2013 [arXiv: 1311.0029].

[2] M. Battaglieri et al., arXiv:1707.04591.

[3] J. Alexander et al., arXiv:1608.08632.

[4] G. W. Bennett et al. (Muon g-2 Collaboration), Phys. Rev. D 73, 072003 (2006).

[5] K. Hagiwara, R. Liao, A. D. Martin, D. Nomura, and T. Teubner, J. Phys. G 38, 085003 (2011).

[6] M. Davier, A. Hoecker, B. Malaescu, and Z. Zhang, Eur. Phys. J. C 71, 1515 (2011); 72, 1874(E) (2012).

[7] M. Pospelov, Phys. Rev. D 80, 095002 (2009).

[8] G. Mohlabeng, Phys. Rev. D 99, 115001 (2019).

[9] G. Bertone and D. Hooper, Rev. Mod. Phys. 90, 045002 (2018).

[10] P. Agrawal, Z. Chacko, and C. B. Verhaaren, J. High Energy Phys. 08 (2014) 147.

[11] Y. Kahn, G. Krnjaic, N. Tran, and A. Whitbeck, J. High Energy Phys. 09 (2018) 153.
[12] B. Batell, N. Lange, D. McKeen, M. Pospelov, and A. Ritz, Phys. Rev. D 95, 075003 (2017).

[13] M. Reece and L.-T. Wang, J. High Energy Phys. 07 (2009) 051.

[14] M. Ibe, W. Nakano, and M. Suzuki, Phys. Rev. D 95, 055022 (2017).

[15] S. Martellotti, in Proceedings, 12th Conference on the Intersections of Particle and Nuclear Physics (CIPANP 2015): Vail, Colorado, USA, 2015 [arXiv:1510.00172].

[16] J. Grange et al. (Muon g-2 Collaboration), arXiv: 1501.06858 .

[17] T. Mibe, Chin. Phys. C 34, 745 (2010).

[18] C.-Y. Chen, M. Pospelov, and Y.-M. Zhong, Phys. Rev. D 95, 115005 (2017).

[19] Y. Kaneta and T. Shimomura, Prog. Theor. Exp. Phys. 05, 053B04 (2017).

[20] P. Abbon et al. (COMPASS Collaboration), Nucl. Instrum. Methods Phys. Res., Sect. A 577, 455 (2007).

[21] S. N. Gninenko, N. V. Krasnikov, and V. A. Matveev, Phys. Rev. D 91, 095015 (2015).

[22] E. Cortina Gil et al., J. Instrum. 12 P05025 (2017).

[23] M. Escudero, D. Hooper, G. Krnjaic, and M. Pierre, J. High Energy Phys. 03 (2019) 071.

[24] X.-G. He, G. C. Joshi, H. Lew, and R. R. Volkas, Phys. Rev. D 44, 2118 (1991).

[25] M. Pospelov and J. Pradler, Annu. Rev. Nucl. Part. Sci. 60, 539 (2010).

[26] A. Kamada and H.-B. Yu, Phys. Rev. D 92, 113004 (2015).

[27] N. Aghanim etal. (Planck Collaboration), arXiv:1807.06209.

[28] See Supplemental Material at http://link.aps.org/ supplemental/10.1103/PhysRevLett.124.041802 for calculations of matrix elements and additional details about our analysis.

[29] C. E. Carlson and B. C. Rislow, Phys. Rev. D 86, 035013 (2012).

[30] E. Cortina Gil et al. (NA62 Collaboration), Phys. Lett. B 791, 156 (2019).

[31] E. Cortina Gil et al. (NA62 Collaboration), Phys. Lett. B 778, 137 (2018).

[32] C.-Y. Chen, H. Davoudiasl, W. J. Marciano, and C. Zhang, Phys. Rev. D 93, 035006 (2016).

[33] B. Batell, A. Freitas, A. Ismail, and D. McKeen, Phys. Rev. D 98, 055026 (2018).

[34] G. Krnjaic, Phys. Rev. D 94, 073009 (2016).

[35] J. P. Lees et al. (BABAR Collaboration), Phys. Rev. D 94, 011102 (2016).

[36] D. Geiregat et al. (CHARM-II Collaboration), Phys. Lett. B 245, 271 (1990).

[37] W. Altmannshofer, S. Gori, M. Pospelov, and I. Yavin, Phys. Rev. Lett. 113, 091801 (2014).

[38] S. R. Mishra et al. (CCFR Collaboration), Phys. Rev. Lett. 66, 3117 (1991).

[39] G. Bellini et al., Phys. Rev. Lett. 107, 141302 (2011).

[40] R. Harnik, J. Kopp, and P. A. N. Machado, J. Cosmol. Astropart. Phys. 07 (2012) 026.

[41] A. Berlin, D. Hooper, G. Krnjaic, and S. D. McDermott, Phys. Rev. Lett. 121, 011102 (2018).

[42] L. Marsicano, M. Battaglieri, A. Celentano, R. De Vita, and Y.-M. Zhong, Phys. Rev. D 98, 115022 (2018).

[43] M. J. Dolan, T. Ferber, C. Hearty, F. Kahlhoefer, and K. Schmidt-Hoberg, J. High Energy Phys. 12 (2017) 094. 
[44] C.-Y. Chen, J. Kozaczuk, and Y.-M. Zhong, J. High Energy Phys. 10 (2018) 154.

[45] $L_{i}(S)=\left[\left(S \epsilon_{S i}+B_{i}\right)^{D_{i}} / D_{i} !\right] e^{-\left(S \epsilon_{S i}+B_{i}\right)}$, where $D_{i}, B_{i}$, and $\epsilon_{S i}$ are data, background, and signal fraction in each bin. The maximum likelihood estimator is $\hat{S}=0$ under the assumptions behind our projections, $D_{i}=B_{i}$.

[46] J. Bijnens, G. Ecker, and J. Gasser, Nucl. Phys. B396, 81 (1993).

[47] M. S. Atiya, I. H. Chiang, J. S. Frank, J. S. Haggerty, M. M. Ito et al., Phys. Rev. Lett. 63, 2177 (1989).
[48] J. Alwall, M. Herquet, F. Maltoni, O. Mattelaer, and T. Stelzer, J. High Energy Phys. 06 (2011) 128.

[49] J. Alwall, R. Frederix, S. Frixione, V. Hirschi, F. Maltoni, O. Mattelaer, H. S. Shao, T. Stelzer, P. Torrielli, and M. Zaro, J. High Energy Phys. 07 (2014) 079.

[50] M. Battaglieri et al. (BDX Collaboration), arXiv:1712.01518.

[51] M. Battaglieri et al. (BDX Collaboration), arXiv:1607.01390.

[52] A. Berlin, N. Blinov, G. Krnjaic, P. Schuster, and N. Toro, Phys. Rev. D 99, 075001 (2019).

[53] T. Akesson et al. (LDMX Collaboration), arXiv:1808.05219. 